\title{
Pap smears Findings of Opportunistic Screening among Reproductive and Postmenopausal Women in Tamil Nadu, India
}

\author{
Kanmani Devi $\mathbf{M}^{*}$ \\ Karpagam Faculty of Medical Science and Research
}

DOI: $\underline{10.36348 / \mathrm{sjpm} .2019 . \mathrm{v} 04 \mathrm{i} 11.015}$

| Received: 20.11.2019 | Accepted: 27.11.2019 | Published: 30.11 .2019

*Corresponding author: Dr. M. Kanmani Devi M.D

\section{Abstract}

Globally cervical cancers affect about $15 \%$ of the women and are the second most common cancer among female population. A dramatic reduction has been observed in the incidence and mortality of cervical cancer after the introduction of Pap test. This study was carried out to assess the role of pap test in cervical cytology and to determine the prevalence of various lesions. This cross sectional study was carried out among 400 patients who attended Gynaecology. Out Patient department. Smears were taken from female patients in the age group of 30-60years with complaints like frothy white discharge, post-coital bleeding, intermenstrual bleeding, dyspaerunia and lower abdominal pain. After fixation in $95 \%$ alcohol and staining, each smear was carefully examined. The cytological smears were made by staining under Papanicolaou's technique and interpreted using new 2001 Bethesda system. Although majority of the smears were negative for any intraepithelial lesion or malignancy, $(87.3 \%)$, inflammation was present in $69.7 \%$ of the smears and $62.5 \%$ showed non specifc inflammation. In addition, 28(7.0\%) showed candida infection and $1(0.25 \%)$ had evidence of Trichomonas infection. Pap test continues to be an essential screening tool to detect early cervical lesions. In addition, it pap test is useful in highlighting the significance of screening test in women of both reproductive and postmenopausal age groups.

Keywords: Cervical cancers, Carcinoma in situ, inflammation, Pap smear, Screening, Bethesda system.

Copyright @ 2019: This is an open-access article distributed under the terms of the Creative Commons Attribution license which permits unrestricted use, distribution, and reproduction in any medium for non-commercial use (NonCommercial, or CC-BY-NC) provided the original author and sources are credited.

\section{INTRODUCTION}

Cervical cancer is one of the most common and leading cancers in the world among the women population, being second next to breast cancers. Studies have shown that around $86 \%$ of all the deaths due to cervical cancer occur in low and middle income countries [1]. Cervical cancer, if detected early can be prevented and the impact and the complications of the cancer can be minimized. However about $50 \%$ of the patients who are diagnosed with cervical cancer die of the disease within five years. In India, the age standardized incidence of cervical cancer is $22 \%$ which is highest among the Asian countries [2].

Majority of the cases of cervical cancer are usually attributed to infectious origin. The key organism causing cervical cancer is Human Papilloma Various (HPV). Although there are several high risk sub types of HPV identified, HPV type 16 and 18 are responsible for about $70 \%$ of all the cervical cancers [3]. In addition to HPV infections, several other factors including smoking, nutritional deficiency, genetic susceptibility and use of hormonal contraceptives are potential risk factors for cervical carcinoma. The progression from normal cervical mucosa to malignancy ranges from inflammation to infiltrative carcinoma of adeno carcinoma and squamous carcinoma types and also carcinoma in-situ. Detection of carcinoma in advanced stages which involve nodal infiltration and distant metastasis, in addition to local widespread growth results in poor prognosis. Similarly, age at diagnosis was considered as significant predictor of the prognosis. Majority of the cancers were being detected at later age beyond 50 years and were associated with poor survival outcomes. However when the cancers are detected earlier the chances of long term survival and better cure are higher.

Considering the impact and morbidity associated with cervical cancers, prevention is the main stay for better prognosis. Prevention of cervical cancer involves minimizing the risk of HPV infection in terms of behavioural modification and vaccination. The other essential strategy for prevention of cervical cancer includes screening through papsmear. The main objective of screening is to detect the changes in the cervical mucosa as early as possible and periodically 
monitor the women so as to prevent invasive carcinoma. Screening for cervical cancer involves good quality reporting, prompt diagnostic investigation, early and appropriate treatment and follow up [3]. So far. Several health authorities globally and nationally have framed guidelines and recommendations for cervical cancer screening. One of the key recommendations concens the age group for screening the cancers which has been fixed as age over 65 years. However there is a need for screening younger population in order to detect pre-cancerous lesions at an early stage so as to minimize the impact of advanced malignancies.

Although several technological advancements have been in place including liquid based cytology and computer assisted imaging, the gold standard screening tool for cervical cancer has been pap smear. Despite the fact that India has population registries on the incidence of cervical cancer, there are few studies which have documented the pap smear findings in younger population, especially in developing countries like, India. This study will aid in modifying the recommendations for screening and also help in minimizing cost and invasive management of cervical cancer, in turn reducing the morbidity and mortality of cervical cancer among reproductive age group women.

\section{Objectives}

This study was carried out to estimate the prevalence and distribution of cervical cancer cytological variants through opportunistic screening.

\section{METHODOLOGY}

\section{Study setting and participants}

This cross sectional study was carried out in the Department of Gynaecology and Obstetrics in our tertiary teaching institution for a period of three months. All the patients who visited the outpatient department with complaints of frothy white discharge, post coital bleeding, inter-menstrual bleeding, dyspaerunia or lower abdominal pain were taken up for the study. A total of 400 women in the reproductive age group participated in the study.

\section{Inclusion criteria}

1. Age 30-60 years

2. Individuals with vaginal discharge and bleeding complaints

\section{Exclusion criteria}

1. Known history of cervical cancer

2. Age $<30$ years; $>60$ years

\section{Ethical approval and inform consent}

Approval was obtained from the Institutional Ethics Committee prior to the commencement of the study. Each participant was explained in detail about the study and informed consent was obtained prior to the commencement of data collection.

\section{DATA COLLECTION}

Pap smear was performed by a single trained gynecologist on all the participants. Before taking the smear, we ensured that no local cream or vaginal douches were used. The patient was made to lie down in dorsal lithotomy position and cusco's speculum was introduced through the vagina and cervix was visualized. Using Ayer's spatula with longer end rotating 360 degrees. The cellular material obtained was smeared on a clean glass slide and transferred to the coplin jar containing $95 \%$ ethyl alcohol which was used as a transport media fixative. The smears were fixed in 95\% alcohol. The cytological smears were made by staining under Papanicolaou's technique and interpreted using new 2001 Bethesta system [4].

\section{RESULTS}

Majority of the participants belonged to the age group of 30-40 years $(43.3 \%)$ and white discharge was the major compliant among the study participants $(53.3 \%)$.(Table 1) The categorization of cytodiagnosis is given in table 2. Normal smear was seen in $17.5 \%$ of the participants while nonspecific inflammation was most common presentation (62.5\%). Among the infiltrative malignancy variants low grade squamous intraepithelial lesion (LSIL) was present in 3\% while squamous cell carcinoma and high grade intraepithelial lesion (HSIL) were present $1 \%$ each. Out of the inflammatory smears, candidal infection $28(7.0 \%)$ was the most predominant type of infection followed by trichomonas infection. While LSIL exhibited koilocytic atypia in majority of the smears, HSIL showed severe dyskeratotic cells with irregular hyper chromatic nuclei with coarsely clumped chromatin. The mean age of cases with LSIL was 32 years while the same with HSIL was 41 years while invasive carcinoma was detected among the age group of 45 - 55 years.

\section{DISCUSSION}

Cervical cytology is currently widely used as the most effective cancer screening modality. WHO(1992) recommended screening every women once in her life time at 40 years. Pap smear examination is widely accepted screening method as it meets both test and disease criteria. In countries like india with predominant rural population with low socioeconomic status, marriage at an early age and poor medical facility predispose the women of the reproductive age group with risk of cervical cancers. In our study mean age of patients with LSIL was 32.5years and those with HSIL and invasive carcinoma were 41-58years respectively. Elhakeem et al. also recorded a progressive increase in development of LSIL to invasive carcinoma with increasing age [5].

LSIL had a peak between 25-35years, HSIL between 36-45years and invasive carcinoma had peak incidence in age grow 50-59 years. Afrakhteh et al found mean age of patients with LSIL, HSIL and invasive cancer to be $37.7,41.7$ and 54.5 respectively 
[6]. The results are in concordance with present study. Khattak et al found that LSIL and HSIL on cytology to be more prevalent in patients who started sexual activity before 20years of age.[7] In present series the prevalence of ASCUS was $0.5 \%$, while SIL was $4 \%$ out of which LSIL was $3 \%$ and HSIL was $1 \%$ and invasive carcinoma was $1.5 \%$. The results are comparable to those obtained by Patel et al and Anuradha and Sinha et al. $[8,9]$.

Although the incidence of invasive cervical carcinoma in our study population is negligible, the population based the registries have documented high rate of incidence in India as against the world's age standardized incidence rate. This could be attributed to several socioeconomic factors including lack of literacy, low socio economic status, early marriages, early child birth and poor hygienic conditions predisposing to an increased risk of sexually transmitted diseases and other infections. Recent trends have shown a decrease in the incidence of the cervical cancer in India, but the decrease is minimal. Moreover there has been a considerable variation in the regional spread of the diseases and very small proportion is diagnosed at a localized stage.

It is disturbing to note that there is no organized mass screening program for early detection of cervical cancer prevalent being practised in India. This is largely attributed to the lack of coordination between organisations and ineffective participation from the public. Therefore considering inadequacies in organising an effective screening program, the postulated decrease in the incidence may be due to under reporting or mal reporting of incidences. Further the 5 year age standardized incidence of cervical cancer is significantly higher in India compared to other countries. This may be attributed to poor socio economic status which indirectly contributes to accessibility and affordability to quality tertiary care. This is evidenced by huge variations between the urban and rural data [10].

\section{CONCLUSION}

The morbidity and mortality associated with cervical cancer can be significantly reduced with a comprehensive large scale mass screening strategy. This screening can be effectively implemented through Pap smear examination which requires adequate infrastructure for cytological examination and staining with aseptic precautions. In developing country like India, it is important that the public health infrastructure works in tandem with doctors and public to ensure that every woman in the reproductive age group is being accounted for and is being addressed for cervical cancer adequately. Considering the advantages of cervical cytology using Pap smear, it is high time that the screening program is being implemented as a routine practice in India.

\section{REFERENCES}

1. Yeole, B. B., Kumar, A. V. R., Kurkure, A., \& Sunny, L. (2004). Population-based survival from cancers of breast, cervix and ovary in women in Mumbai, India. Asian Pacific Journal of Cancer Prevention, 5(3), 308-315.

2. ICO Information Centre on HPV and cancer (Summary Report 2014-08-22). (2014). Human Papillomavirus and Related Diseases in India.

3. Denny, L., Sankaranarayanan, R. (2006). Secondary prevention of cervical cancer. International Journal of Gynecology and Obstetrics, 94 Suppl 1, S65-S70.

4. Nayar, R., \& Wilbur, D. C. (Eds.). (2015). The Bethesda system for reporting cervical cytology: definitions, criteria, and explanatory notes. Springer.

5. Elhakeem, H. A., Al-Ghamdi, A. S., \& AlMaghrabi, J. A. (2005). Cytopathological pattern of cervical Pap smear according to the Bethesda system in Southwestern Saudi Arabia. Saudi medical journal, 26(4), 588-592.

6. Afrakhteh, M., Khodakarami, N., Moradi, A., Alavi, E., \& Shirazi, F. H. (2007). A study of 13315 papanicolau smear diagnoses in Shohada Hospital. Journal of family and reproductive health, 74-78.

7. Khattak, S. T., Naheed, T., Akhtar, S., \& Jamal, T. (2006). Detection of abnormal cervical cytology by pap smears. Gomal Journal of Medical Sciences, 4(2).

8. Patel, T. S., Bhullar, C., Bansal, R., \& Patel, S. M. (2004). Interpreting epithelial cell abnormalities detected during cervical smear screening--a cytohistologic approach. European journal of gynaecological oncology, 25(6), 725728.

9. Sinha, A. (2005). Conventional pap-smear screening for human papilloma virus related lesions of the cervix. Journal of Cytology, 22(1), 32.

10. Bobdey, S., Sathwara, J., Jain, A., \& Balasubramaniam, G. (2016). Burden of cervical cancer and role of screening in India. Indian journal of medical and paediatric oncology: official journal of Indian Society of Medical \& Paediatric Oncology, 37(4), 278. 